Rev. Inst. Flor. v. 28 n. 2 p. 111-117 dez. 2016

http://dx.doi.org/10.24278/2178-5031.201628203

ISSN impresso 0103-2674/on-line 2178-5031

\title{
FUNÇÕES DE DENSIDADE PROBABILÍSTICA PARA PREDIÇÃO DA DISTRIBUIÇÃO DIAMÉTRICA EM PLANTIOS DE EUCALIPTO EM FUNÇÃO DO ESPAÇAMENTO ${ }^{1}$

\author{
PROBABILITY DENSITY FUNCTIONS FOR PREDICTION \\ OF DIAMETER DISTRIBUTION IN EUCALYPTUS PLANTATIONS \\ IN SPACING FUNCTION
}

\author{
Thomas SCHRODER ${ }^{2}$; Anna Paula Lora ZIMMERMANN²,3; \\ Frederico Dimas FLEIG ${ }^{2}$; Victor Carlos DOMINGOS NETO ${ }^{2}$
}

\begin{abstract}
RESUMO - Buscando uma alternativa para preencher a lacuna existente entre variáveis de povoamentos e variáveis de árvore individuais para estimação de diferentes sortimentos de madeira, foram medidos plantios de Eucalyptus dunnii Maiden em São Francisco de Assis, RS. Quatro diferentes espaçamentos foram analisados e verificado o ajuste das funções de distribuição de probabilidade Normal, Log-Normal, Gama e Weibull (dois parâmetros). Os espaçamentos medidos foram: 0,87 m x 1,75 m; $1,75 \mathrm{~m}$ x 1,75 m; $1,75 \mathrm{~m}$ x 3,5 m e 3,5 m x 3,5 m. As funções foram avaliadas pelo coeficiente de aderência de Kolmogorov-Smirnov e pela soma dos escores. Os coeficientes foram ajustados em função do espaçamento inicial e avaliados pelo coeficiente de determinação ajustado. A função que melhor explicou a distribuição diamétrica dos dados foi a Log-Normal. Esta apresentou bom ajuste para os seus coeficientes, e de forma simples, com a utilização de apenas uma variável, facilmente determinada a campo.
\end{abstract}

Palavras-chave: predição da produção; Eucalyptus dunii; Weibull; sortimentos de madeira.

\begin{abstract}
Seeking an alternative to fill the gap between stands variables and individual tree variables for prediction of the wood assortments, Eucalyptus dunni Maiden plantations in São Francisco de Assis, RS, Brazil was measured. Four different spacings were analyzed and the adjusting the probability distribution functions Normal, Log Normal, Gamma, and Weibull (two parameters) were verified. The spacing measured were $0.87 \mathrm{~m} \times 1.75 \mathrm{~m} ; 1.75 \mathrm{~m} \times 1.75 \mathrm{~m} ; 1.75 \mathrm{~m} \times 3.5 \mathrm{~m}$ and $3.5 \times 3.5 \mathrm{~m}$. The functions were evaluated by Kolmogorov-Smirnorv adherence test and by the sum of scores. The coefficient were adjusted by initial spacing and evaluated by adjusted coefficient determination. The function with better adjusting was Log-Normal. The function that best explains the diameter distribution of the data was Log-Normal. This presented a good fit for its coefficients, and in a simple way, with the use of only one variable, easily determined in the field.
\end{abstract}

Keywords: prediction of production; Eucalyptus dunni; Weibull; wood assortment.

${ }^{1}$ Recebido para análise em 03.05.2016. Aceito para publicação em 06.09.2016.

2Programa de Pós-graduação em Engenharia Florestal, Universidade Federal de Santa Maria, Avenida Roraima, 1000, 97195-000, Santa Maria, RS, Brasil.

${ }^{3}$ Autor para correspondência: Anna Paula Lora Zimmermann - zimmermann-a@hotmail.com 


\section{INTRODUÇÃO}

Gram-Charlier, Beta e Weibull (Bailey e Dell, 1973; Hafley e Schreuder, 1977; Cunha Neto et al, 1994; Maltamo et al., 1995; Barra et al., 2005; Palahí et al., 2007; Abbasi et al., 2008).

Existem, essencialmente, quatro método espécie com capacilade de resistencia sendo bastate tilizada en planis en locis de grande alitude e latifde. Sun madira pas de grande altitude e latitude. Sua madeira possui características semelhantes ao E. grandis, de boa
estabilidade dimensional e outras características desejáveis comercialmente (Severo, 2009).

A predição da produção florestal em termos volumétricos é o que permite a avaliação da viabilidade e rentabilidade de projetos florestais. No caso de povoamentos manejados em densidade completa, para fins de biomassa para energia apenas o volume comercial total a ser produzido deve ser predito, pois a utilização da madeira produzida é relativamente pouco criteriosa No entanto, em florestas manejadas para produção de múltiplos produtos, uma variedade de diâmetros e comprimentos de toras deve ser levad em consideração de acordo com a dimensão desejada do produto final, como, por exemplo, energia, celulose, escora e serraria, entre outros.

A maioria dos modelos de predição florestal baseia-se em variáveis relacionadas ao povoamento, como área basal e altura dominante. Os diferentes sortimentos de madeira produzidos são estimados através de funções de afilamento, que se baseiam no diâmetro e altura da árvore individual. Essa lacuna entre variáveis de povoamento variáveis de árvores individuais é preenchida po funções de predição de distribuição diamétrica.

Diversas funções de densidade probabilística podem ser utilizadas par predição da distribuição diamétrica, sendo estas: Normal, Log-Normal, Gama, Johnson's SB,

Tabela 1. Descrição dos plantios de E. dunnii, São Francisco de Assis - RS. Table 1. Description of E. dunnii plantations, São Francisco de Assis - RS

\begin{tabular}{cccc}
\hline Tratamento & $\mathrm{N}^{\circ}$ de árvores amostradas & Espaçamento $(\mathrm{m})$ & Espaço Vital $\left(\mathrm{m}^{2}\right)$ \\
\hline $\mathrm{T} 1$ & 41 & $0,87 \times 1,75$ & 1,53 \\
$\mathrm{~T} 2$ & 58 & $1,75 \times 1,75$ & 3,06 \\
$\mathrm{~T} 3$ & 61 & $1,75 \times 3,5$ & 6,13 \\
$\mathrm{~T} 4$ & 70 & $3,5 \times 3,5$ & 12,25 \\
\hline
\end{tabular}

Foram testadas as funções Normal, Log-Normal, Gama e Weibull de dois parâmetros, para cada densidade de plantio. $\mathrm{O}$ ajuste das funções foi avaliado através da média dos espaçamentos, pelo coeficiente de aderência de espaçamentos, pelo coeficiente de aderência de
Kolmogorov-Smirnov para cada uma das funções (Binoti et al, 2013). A escolha da melhor função foi feita através da soma dos escores.

Posteriormente, foram ajustadas todas as funções para cada uma das repetições do experimento. Os coeficientes obtidos foram reestimados em função do espaçamento inicial em suas formas aritmética, quadrática, raiz, logaritmo natural e inversa. $\mathrm{O}$ modelo foi desenvolvido através do procedimento stepwise e, seu ajuste foi avaliado em funcão do coeficiente de determinação ajustado.

Os procedimentos estatísticos foram realizados com o software $\mathrm{R}$ ( $\mathrm{R}$ Core Team, 2013) através do pacote fitditrplus (Delignette-Mulle et al., 2012).

\section{RESULTADOS E DISCUSSÃO}

As funções de densidade probabilística mostraram comportamentos distintos de ajuste no diferentes espaçamentos iniciais (Tabela 2). Todo os ajustes foram significativos à probabilidade de $5 \%$. A função Log-Normal apresentou o melhor ajuste médio entre os diferentes espaçamentos, sendo seguida pela função Normal, que apresentou menor variação de ajuste.

Tabela 2. Valor do teste de aderência de Kolmogorov-Smirnov para o ajuste das funçooes de densidade probabilística avaliadas em diferentes espaçamentos de E. dunnii, em São Francisco de Assis - RS.

Table 2. Value of Kolmogorov-Smirnov adhesion test for the adjustment of the probabilistic density functions evaluated in different spacing of $E$. dunnii, São Francisco de Assis - RS.

\begin{tabular}{ccccc}
\hline \multirow{2}{*}{ Tratamento } & \multicolumn{3}{c}{ Função } \\
\cline { 2 - 5 } & Weibull & Gama & Normal & Log-Normal \\
\hline T1 & 0,1457 & 0,1145 & 0,0754 & 0,0543 \\
T2 & 0,0590 & 0,0689 & 0,0832 & 0,0895 \\
T3 & 0,1130 & 0,0929 & 0,0615 & 0,0444 \\
T4 & 0,0685 & 0,0673 & 0,0582 & 0,0521 \\
\hline Média & 0,0965 & 0,0859 & 0,0696 & 0,0601 \\
Desvio Padrão & 0,0403 & 0,0224 & 0,0118 & 0,0201 \\
\hline
\end{tabular}

A função de Weibull, conhecida na pelo espaçamento inicial de plantio (Tabela 1). Os dados foram obtidos quatro anos após a mplantação do experimento, totalizando uma área mostrada de 1,96 hectares. literatura pela sua flexibilidade (Cañadas et al., 2002; Pretzsch, 2010), apresentou o menor ajuste médio e a maior variação entre as densidades de plantio, além do pior resultado para o espaçamento mai adensado. Isso pode ser explicado devido ao fato de esse teste ser sensível ao número de diâmetros utilizados (Teo et al., 2011).

A soma dos escores do teste de aderência mostrou que a função que mais se adaptou à distribuição diamétrica foi função Log-Normal, seguida pela Normal (Tabela 3 ). No entanto, no tratamento 2 (espaço vital 3,06) o comportamento do ajuste das funções diferiu do padrão observado nos demais tratamentos. Nesse tratamento e no tratamento 4 (espaço vital 12.25), as diferenças no ajuste foram menores, demonstrando que essas distribuições diamétricas apresentam comportamento intermediário entre as distribuições teóricas das funções de densidade probabilística.

Observa-se, na Figura 1, a tendência natural de maior frequência de diâmetros pequeno em espaçamentos mais adensados e maiore diâmetros médios em espaçamentos mais amplos. Além disso, é possível observar a curtose negativa da distribuição dos dados. 
Tabela 3. Escores do ajuste das funções de densidade probabilística para diferentes tratamentos de espaçamento de E. dunnii realizados em São Francisco de Assis - RS.

Table 3. Scores of the adjustment of the probabilistic density functions for different spacing treatments of $E$. dunni performed in São Francisco de Assis - RS.

\begin{tabular}{ccccc}
\hline \multirow{2}{*}{ Tratamento } & \multicolumn{4}{c}{ Função de Distribuição de Probabilidade } \\
\cline { 2 - 5 } & Weibull & Gama & Normal & Log-Normal \\
\hline T1 & 4 & 3 & 2 & 1 \\
T2 & 1 & 2 & 3 & 4 \\
T3 & 4 & 3 & 2 & 1 \\
T4 & 4 & 3 & 2 & 1 \\
\hline Soma & 13 & 11 & 9 & 7 \\
\hline
\end{tabular}

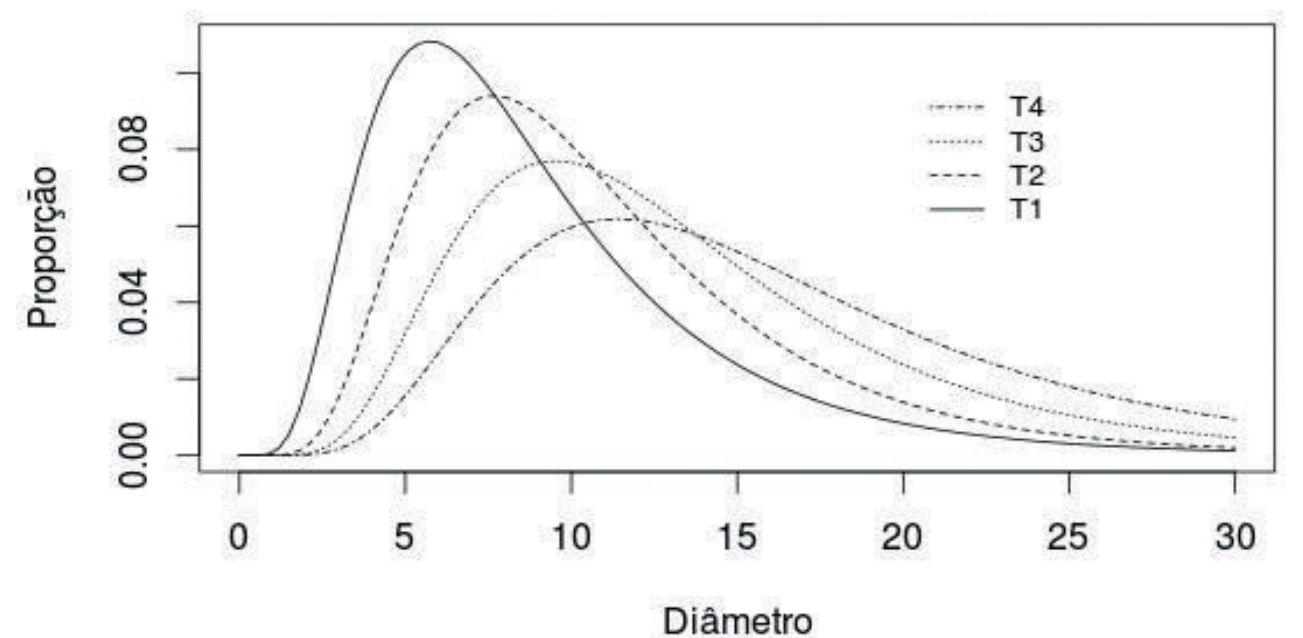

Figura 1. Distribuições diamétricas ajustadas pela função de densidade probabilística Log-Normal para diferentes densidades de plantio de E. dunnii, em São Francisco de Assis - RS

Figure 1. Diameter distributions adjusted by lognormal probability density function for different planting densities of E. dunnii, in São Francisco de Assis - RS.

Os modelos desenvolvidos para predição dos coeficientes das diferentes funções de densidade probabilística mostraram ajustes bastante distintos (Tabela 4). De forma geral, as melhores estimativas de coeficientes foran alcançadas quando a distribuição Norma foi utilizada. A função de Weibull apresentou resultados intermediários e as demais não apresentaram ajustes desejáveis.

Existe, na literatura, um esforço no sentido da predição dos coeficientes para predição da distribuição diamétrica (Cao, 2004

Rev. Inst. Flor. v. 28 n . 2 p. $111-117$ dez. 2016
Tabela 4. Funções de densidade probabilística ajustada e reestimada para distribuição diamétrica de $E$. dunnii em diferentes densidades de plantio, São Francisco de Assis - RS.

Table 4. Probability density functions adjusted and estimated for diametric distribution of $E$. dunnii in different planting densities, São Francisco de Assis - RS.

\begin{tabular}{cccc}
\hline Distribuição & Coeficiente & Modelo & $\mathrm{R}_{\mathrm{aji}}^{2}$ \\
\hline \multirow{2}{*}{ Weibull } & Forma & $1,76974+0,38402 * \sqrt{E s p}$ & 0,6291 \\
& Escala & $6,0757+3,4982 * \sqrt{E s p}$ & 0,9119 \\
\multirow{2}{*}{ Gama } & Forma & $3,2737+0,7851 * \sqrt{E s p}$ & 0,2362 \\
& Taxa & $0,462189-0,008724 * E s p$ & 0,2594 \\
\multirow{2}{*}{ Normal } & Média & $5,2298+3,1879 * \sqrt{E s p}$ & 0,9098 \\
& Desvio Padrão & $3,958186+0,292139 * E s p+0,011032 * E s p^{2}$ & 0,9024 \\
& Log - Média & $1,42813+0,60174 * \sqrt{E s p}-0,06884 * E s p$ & 0,8810 \\
& Log-Normal & 0,49464 & 0 \\
\hline
\end{tabular}

Em que: Esp = espaço vital por árvore expresso em metros quadrados.

In which: Esp = vital space per tree in square metres.
Weibul
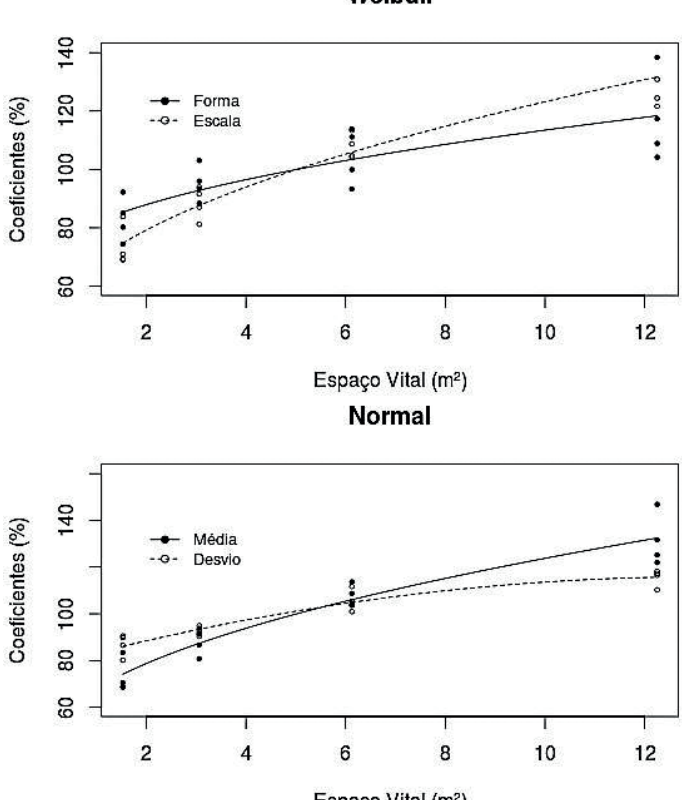

Gama
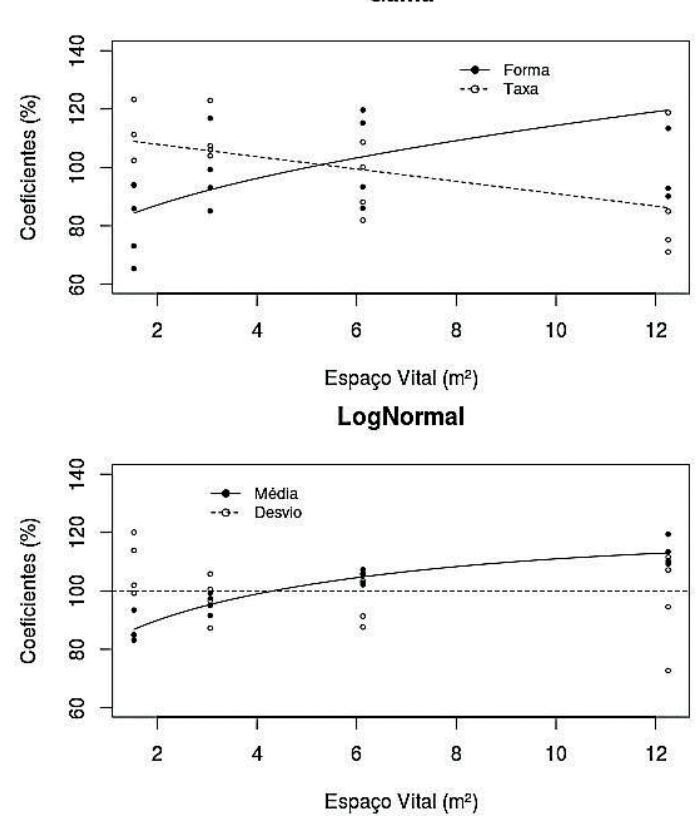

Figura 2. Valores observados e ajustados das funções de densidade probabilística ajustadas para diferentes densidades de plantio de E. dunnii, São Francisco de Assis - RS.

Figure 2. Observed and ajusted values of probability density functions for different planting densities of E. dunnii, São Francisco de Assis - RS.
2006; Schneider, 2012). No entanto, encontrados demonstram que outras funcões são capazes de estimar a distribuição diamétrica ajustes semelhantes e inclusive melhores disso, a estimativa dos coeficientes, neste caso, ser feita de forma simples, em função apenas espaço inicial disponível para cada árvore (Figura 2). 


\section{CONCLUSÃO}

A distribuição Log-Normal, apesar de possuir melhor aderência, não apresento ajuste significativo de um dos seus coeficientes. Dessa forma, no presente estudo, a distribuição normal foi a que melhor se adaptou estimativa da distribuição diamétrica, visto que apresentou boa aderência pelo teste Kolmogorov-Smirnov e que foi possível encontrar um bom ajuste para os seus coeficientes utilizando-se apenas as variáveis de espaçamento.

\section{REFERÊNCIAS BIBLIOGRÁFICAS}

ABBASI, B.; RABELO, L.; HOSSEIN KOUCHACK, M. Estimating parameters of the three-parameter Weibull distribution using a neural network. European Journal of Industrial Engineering v. 2, n. 4, p. 428-445, 2008.

BAILEY, R.L.; DELL, T.R. Quantifying diameter distributions with the Weibull function. Forest Science, v. 19, n. 2, p. 97-104, 1973.

BARRA, O.S.V. et al. Proposta metodológica para o ajuste ótimo da distribuição diamétrica Weibull 3P. Floresta, v. 34, n. 3, p. 387-393, 2005

BINOTI, D.H.B.; BINOTI, M.L.M.D.S.; LEITE, H.G. Utilização da função pearson tipo $\mathrm{V}$, Weibull e hiperbólica para modelagem da distribuição de diâmetros. Pesquisa Florestal Brasileira, v. 33, n. 75 , p. $279-286$, jul./set. 2013

CAÑADAS, N.; MONTERO GONZÁLEZ, G GARCÍA GÜEMES, C. Modelización de la distribución diamétrica de lãs masas de "Pinus pinea" L. de Valladolid (España) mediante la función Weibull. Investigación Agraria Sistemas y Recursos Forestales, v. 11, n. 2, p. $263-282,2002$

CAO, Q.V. Predicting parameters of a Weibull function for modeling diameter distribution. Forest Science, v. 50, n. 5, p. 682-685, 2004.
CUNHA NETO, F. da C. et al. Modelo para predição da produção por classe de diâmetro para Eucalyptus grandis. Cerne, v. 1, n. 1 p. 108-122, 1994

DELIGNETTE-MULLER, M.L. et al. Fitdistrplus: help to fit of a parametric distribution to non-censored or censored data. $R$ package version $0.1-3,2012$

EMPRESA BRASILEIRA DE PESQUISA AGROPECUÁRIA - EMBRAPA. Centro Naciona de Pesquisa de Solos. Sistema brasileiro de classificação de solos. Rio de Janeiro: Embrapa Solos, 2006. p. 306.

HAFLEY, W.L.; SCHREUDER, H.T. Statistical distributions for fitting diameter and height data in even-aged stands Canadian Journal of Forest Research, v. 7, n. 3, p. 481-487, 1977.

KLUGMAN, S.A.; PANJER, H.H.; WILLMOT, G.E. Loss models: from data to decisions. 4th ed. [s.l.] John Wiley \& Sons, 2012. 536 p.

LEITE, H.; NOGUEIRA, G.; MOREIRA, A. Efeito do espaçamento e da idade sobre variáveis de povoamentos de Pinus taeda L. Revista Árvore, v. 30, n. 4 , p. $603-612,2006$

et al. Comparação entre predição e projeção da distribuição de diâmetros de povoamentos de eucalipto submetidos a desbastes. Revista Arvore, v. 37, n. 2, p. 321-328, 2013

MALTAMO, M. PUUMALAINEN, J.; PÄIVINEN, R. Comparison of beta and Weibull functions for modelling basal area diameter distribution in stands of Pinus sylvestris and Picea abies. candinavian Journal of Forest Research, v. 10 n. 1-4, p. 284-295, 1995.

MORENO, J.A. Clima do Rio Grande do Su Porto Alegre: Secretaria da Agricultura, Diretoria da Agricultura, Diretoria de Terras e Colonização, Secção de Geografia, 1961. p. 42.
PALAHI, M. et al. Comparison of beta, Johnson's SB, Weibull and truncated Weibull functions for modeling the diameter distribution of forest stands in Catalonia (north-east of Spain). European Journal of Forest Research, v. 126, n. 4, p. 563-571, 2007.

PRETZSCH, H. Forest dynamics, growth, and yield. Berlin: Springer, 2010. p. 671

R CORE TEAM. R: a language and environment for statistical computing [Internet]. Vienna: R Foundation for Statistical Computing, 2013.

SCHNEIDER, P.S.P. Estrutura bioeconômica da produção no manejo da densidade de Pinus taeda L na região do Planalto Catarinense, Brasil. 2012 188 f Tese (Doutorado

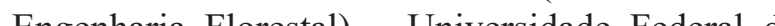
Santa Maria Santa Maria.

SEVERO, E. Qualidade da secagem de madeira serrada de Eucalyptus dunnii. Ciência Florestal, v. 10, p. $109-124,2009$

TEO, S.J. et al. Dinâmica da distribuição diamétrica de povoamentos de Pinus taeda, na regĩo de Caçar, SC. Pesquisa Florestal Brasileira, v. 31, n. 67, p. 183-192, 2011 